\title{
Ergonomic evaluation of laparoendoscopic single-site surgery ports in a validated laparoscopic training model
}

\author{
Bernadette McCrory ${ }^{\mathrm{a}}$, Bethany R. Lowndes ${ }^{\mathrm{a}}$, Laura M. Wirth ${ }^{\mathrm{a}}$, Adam E. de Laveaga ${ }^{\mathrm{a}}$, Chad A. \\ LaGrange ${ }^{\mathrm{b}}$ and M. Susan Hallbeck ${ }^{\mathrm{a}, *}$ \\ ${ }^{a}$ Department of Industrial and Management Systems Engineering, University of Nebraska-Lincoln, 342 Nebraska \\ Hall, Lincoln, NE 68588-0656, USA. \\ ${ }^{\mathrm{b}}$ Department of Surgery, Section of Urology, University of Nebraska Medical Center, Omaha, NE 68198, USA
}

\begin{abstract}
Although laparoendoscopic single-site surgery (LESS) is feasible among expert laparoscopic surgeons, it poses many technical challenges not seen in conventional laparoscopy (CL). Recent technological advancements in single-incision instrumentation have created more interest and widespread usage of LESS. However, neither LESS nor its novel instrumentation have been thoroughly studied or evaluated using human factors and ergonomics techniques. Consequently, the aim of this study was to compare the physical performance of LESS to CL using a standardized task. Wrist and elbow angular movements, range of motion and physical discomfort were assessed for 24 novice participants. There were no significant differences for physical comfort/discomfort ratings or elbow and wrist flexion/extension range of motion between CL and LESS. However, wrist radial/ulnar range of motion was significantly greater in LESS compared to CL $(p<0.05)$. Additionally, wrist radial/ulnar range of motion was significantly greater using the SILS Port compared to the GelPOINT ( $<<0.05)$. Although further investigation is needed, LESS resulted in greater wrist deviation and range of motion due to the close proximity of the instruments, restrictive nature of the single-port devices, and the need to achieve adequate instrument triangulation and visualization.
\end{abstract}

Keywords: single-incision, laparoscopy, goniometry, human factors, simulation

*Corresponding author. E-mail: hallbeck@unl.edu. 


\section{Introduction}

Laparoendoscopic single-site surgery (LESS), the next advance in minimally invasive surgery (MIS), is a feasible surgical technique performed using a single, small incision typically within the patient's navel [10 $-11,14]$. The surgeon inserts several instruments and a laparoscopic camera into the single incision leaving virtually no surgical scar. LESS is the newest alternative to conventional multi-incision laparoscopic surgery and natural orifice transluminal endoscopic surgery (NOTES) [6-7]. The evolution of LESS primarily occurred due to the recent development of advanced access port technology (i.e., single-port devices) and the technical performance difficulty of NOTES [1, 6-7, 12].

However, LESS poses physical, mental and technical performance challenges unique to this surgical technique [7]. Since all of the instrumentation is inserted through a single incision, the surgeon must contend with instrument collisions, transposed instrument viewing (i.e., the surgeon's right instrument operates on the left side), and an in-line view of the instruments. Similar to conventional laparoscopy, the surgeon must also still contend with a static and nonneutral body posture due to the elongated instruments, elevated monitor positions, multiple foot pedals and operating table height [8-9, 15-16]. Since LESS emerged quite rapidly, surgeons have primarily relied upon conventional laparoscopic instrumentation, which have not been designed or optimized for LESS. Very recently, there has also been an influx of highly complex instrumentation for LESS. Although these novel hand instruments and access ports seem to be aiding LESS surgeons, there have been no published reports on the ergonomics of these devices and their potential effects on surgical performance. As a result, the aim of this study was to compare the physical performance of LESS to conventional laparoscopy.

\section{Materials and methods}

The same participant population, apparatus and procedure were used as previously described in Brown-Clerk et al. [2].

\subsection{Participants}

Twenty-four healthy adults (12 males and 12 females) were recruited to participate in this study. The participants were medical students, undergraduate and graduate students from the local medical center. Participant exclusion criteria included prior surgical experience and experience with the manual skills portion of the Fundamentals of Laparoscopic Surgery (FLS) program. Twenty-two participants were righthand dominant and one male and one female were left hand-dominant. A descriptive summary of the participants is shown in Table 1.

Table 1

Participant Descriptive Summary

\begin{tabular}{|l|c|c|c|}
\hline & $\begin{array}{c}\text { Age } \\
\text { (years) }\end{array}$ & $\begin{array}{c}\text { Weight } \\
(\mathrm{kg})\end{array}$ & $\begin{array}{c}\text { Height } \\
(\mathrm{cm})\end{array}$ \\
\hline & 24.3 & 81.9 & 178 \\
Male & $(2.57)$ & $(12.6)$ & $(12.1)$ \\
\hline & 25.3 & 67.9 & 167 \\
Female & $(5.79)$ & $(18.1)$ & $(8.71)$ \\
\hline & 24.8 & 74.9 & 173 \\
Overall & $(4.41)$ & $(16.9)$ & $(11.7)$ \\
\hline
\end{tabular}

Note: Mean (Standard Deviation)

\subsection{Apparatus}

The experimental set-up consisted of a FLS manual skills trainer, FLS peg transfer task, standard monitor tower (OfficeKart 9802 T-20, Karl Storz, Tuttlingen, Germany) with widescreen LCD HD monitor (56-cm, ViewSonic, Walnut, CA, USA), and a stationary high-speed HD camera (Logitech Quickcam Pro 9000 web camera, Fremont, CA, USA). Two standard length non-locking 5-mm graspers (Auto Suture Endo Dissect, Covidien, Mansfield, MA, USA) were used throughout the study. The trainer was securely positioned on an adjustable height table in front of the monitor tower. The stationary HD camera displayed the task field through the monitor at an approximately 30-degree viewing angle. Both the trainer and monitor were placed in-line with the participant. 


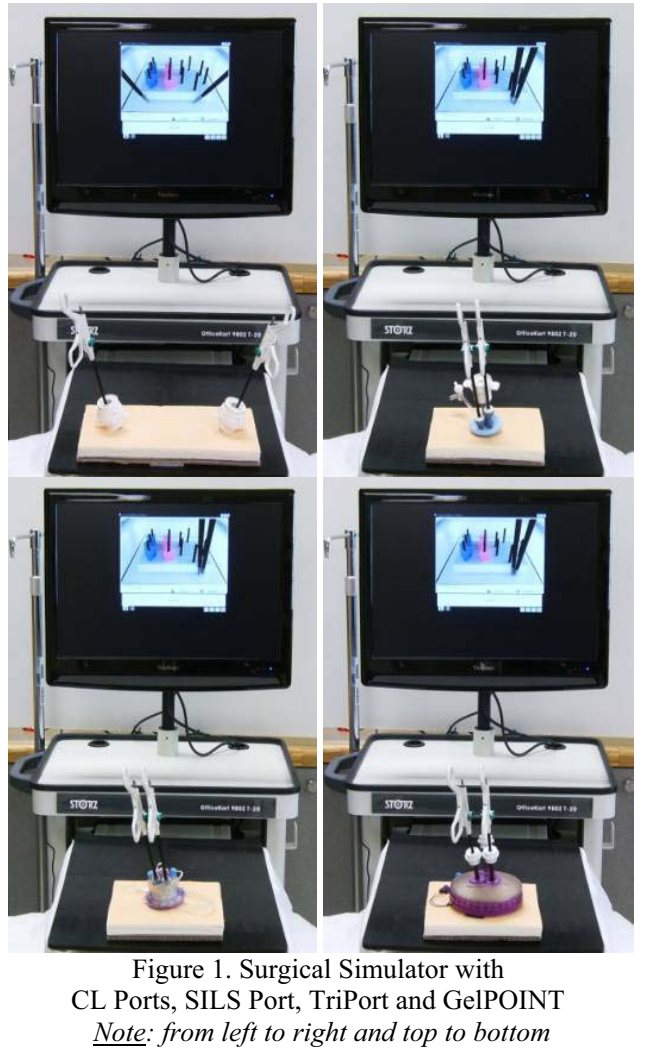

The novel surgical simulator (Figure 1) was adapted from the FLS manual skills trainer for LESS to include a 15-mm synthetic skin interface (LaproAbdominal Pad, Limbs and Things ${ }^{\mathrm{TM}}$, Bristol, UK). The SILS ${ }^{\text {TM }}$ port (Covidien, Mansfield, MA, USA), TriPort ${ }^{\mathrm{TM}}$ Access System (Olympus America Inc., Center Valley, PA, USA), and GelPOINTTM System (Applied Medical, Rancho Santa Margarita, CA, USA) were inserted into an $2.0-\mathrm{cm}$ incision through the synthetic skin. For CL, two standard 12-mm trocars were inserted through a $1.5-\mathrm{cm}$ incision $18-\mathrm{cm}$ apart in the synthetic skin.

\subsection{Task}

The FLS manual skills curriculum consists of five basic laparoscopic surgical tasks, which develop skills such as ambidexterity, depth perception, handeye coordination and controlled movement of instruments [4-5]. The first FLS task, peg transfer, requires the surgical trainee to grasp, lift, transfer and place six small triangle shaped objects on a pegboard starting with the non-dominant hand and transferring to the dominant hand. Once the trainee has repositioned all six objects to the opposite side of the pegboard, the procedure is reversed where the object is grasped with the dominant hand and transferred to the nondominant hand resulting in a total of 12 peg transfers.

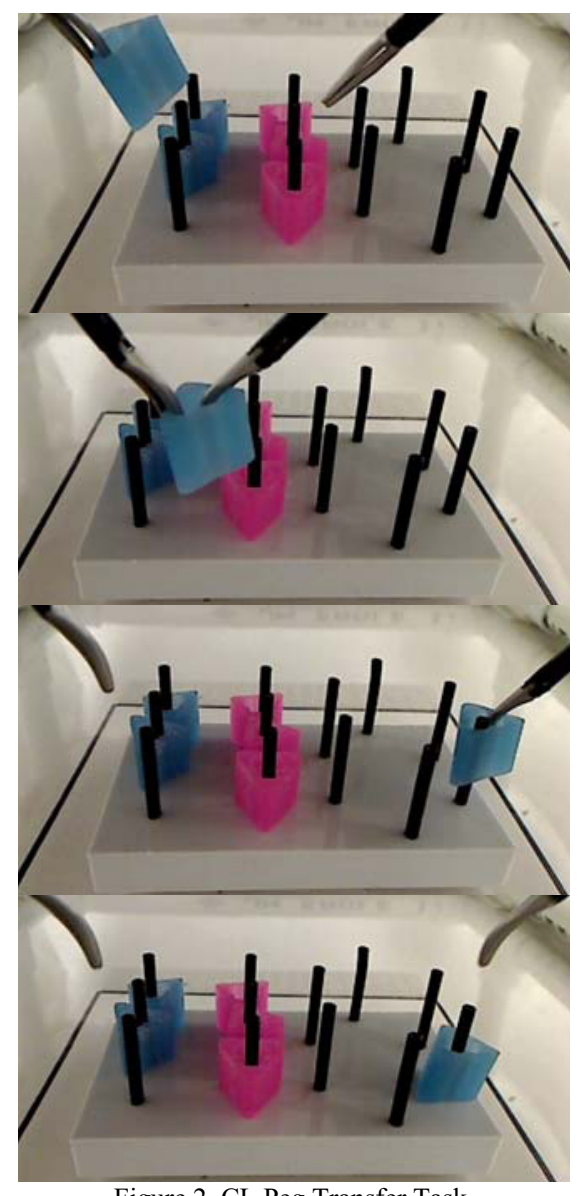

Figure 2. CL Peg Transfer Task

Completion of the peg transfer task in the CL and LESS simulators are shown in Figures 2 and 3, respectively. The same right-hand dominant participant performed the peg transfer task in Figures 2 and 3 beginning with the left (i.e. non-dominant) hand. The starting positions of the pegs are reversed during LESS due to the transposed instrument orientation. As seen in Figure 2, CL task completion is aided through optimal instrument placement that facilitates instrument articulation, triangulation and visualization. In contrast, the location and orientation of the instrumentation in the LESS simulator results in collisions, transposition and an in-line view of the instruments as shown in Figure 3. 


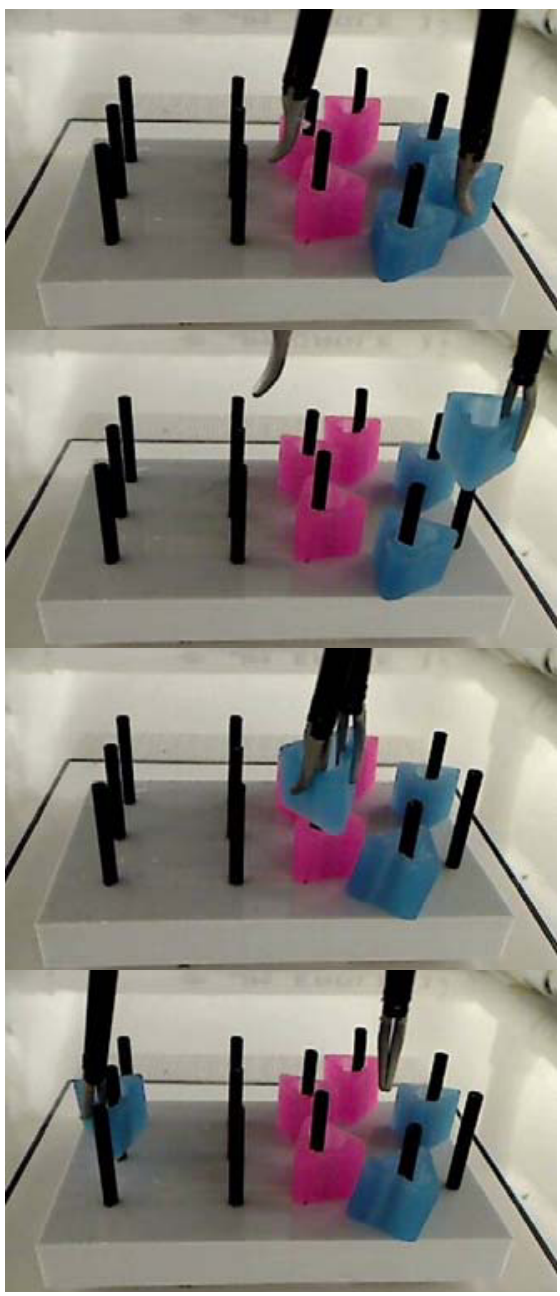

Figure 3. LESS Peg Transfer Task

\subsection{Procedure}

This study was conducted in accordance with local IRB standards and protocols. The experimental procedures were explained to each participant prior to the conduct of the study. Demographic data and study inclusion/exclusion criteria were annotated at the start of the study. Table height was adjusted to each participant's standing elbow height [3], and each participant donned hypoallergenic surgical gloves in a selfselected size. The FLS proctor script was followed whereby the manual skills written instructions, performance guidelines and video demonstration were explained and shown prior to the conduct of the study. The participants were instructed to complete the peg transfer task in accordance with the FLS criteria.

Next, limb angular movements were used to compare conventional laparoscopy and the single-port devices. Limb angular movement was measured us- ing twin-axis electrogoniometers. Each subject's dominant wrist and elbow angular movements were monitored using electrogoniometers (Biometrics Ltd, Gwent, UK) SG65 and SG110, respectively. Each electrogoniometer was applied using medical-grade double-sided adhesive tape. The wrist was monitored in both the flexion-extension and radial-ulnar deviation planes. The elbow was monitored in the flexionextension plane. In accordance with the Goniometer and Torsiomenter Operating Manual (Biometrics Ltd, Copyright 2002) the datum position for each measurement plane was set for each participant in the prescribed neutral joint position. Wrist and elbow angular movements were recorded at a sampling rate of 200/sec using the DataLINK system (Model DLK900, Biometrics Ltd, Gwent, UK) with software version 7.0. The maximum and minimum angular positions for each movement plane were calculated using the DataLINK system software. The included elbow angle was calculated using $0^{\circ}$ as fully flexed and $180^{\circ}$ as fully extended. Range of motion (ROM) was also calculated for each plane by taking the difference between the maximum and minimum angular positions.

Following goniometer placement and calibration, each participant completed a brief hands-on familiarization period of five minutes in the CL setup. Afterwards he or she performed the peg transfer task using CL ports, SILS Port, TriPort, and the GelPOINT with two standard length 5-mm graspers. Each participant completed the task first using CL followed by randomized completion using each of three single-port devices. Due to task length only one task trial was completed for each port resulting in a total of four trials per participant.

A rest period of five minutes was given between each port to minimize fatigue and to administer a short questionnaire. The questionnaire was administered directly following task completion in each of the four ports, and rated the comfort/discomfort of six anatomical regions on a verbally-anchored 6-point Likert scale with a forced choice method.

\subsection{Experimental design}

A full-factorial analysis of variance with blocking on subjects was performed for the dependent variable angular position and range of motion for each movement plane using SAS (V. 9.2). Post-hoc Tukey tests were performed for significant main effects. Nonparametric Kruskal-Wallis' tests were performed for the dependent variable statement rating 
for each anatomic region and instrument maneuverability using MINITAB (V. 14.2). For all statistical tests the independent variable was port (4 levels) and the level of significance was set at 0.05 .

\section{Results}

\subsection{Elbow flexion/extension}

There were no significant differences for the average minimum elbow extension, maximum elbow extension or elbow ROM across ports. As expected elbow angular positioning (i.e. included angle) was relatively static for all ports. Average elbow ROM was the greatest in the TriPort at $38^{\circ}$ and the least in $\mathrm{CL}$ at $28^{\circ}$ as depicted by the dashed line in Figure 4.

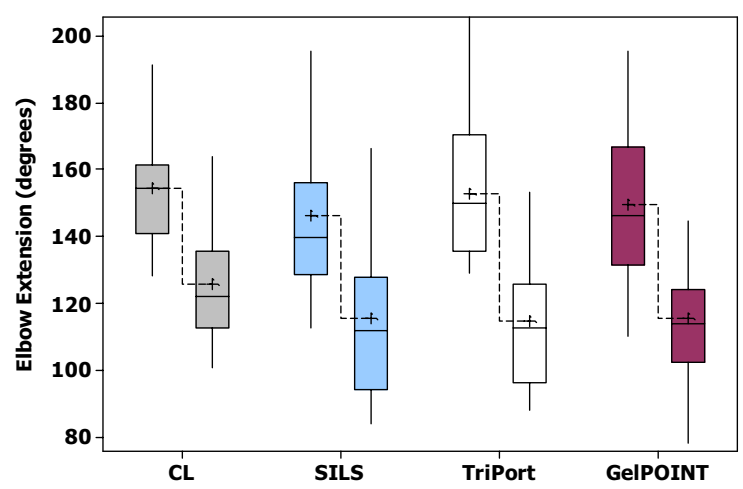

Figure 4. Elbow Flexion/Extension Notes: median horizontal line, mean plus sign, mean ROM dashed line

\subsection{Wrist flexion/extension}

There was no significant difference for the average wrist ROM, which was approximately $70^{\circ}$, across all ports. However, the average maximum flexion and minimum extension were significantly different between CL and both the TriPort and GelPOINT ( $\mathrm{p}<$ 0.05). Specifically, wrist flexion for CL was significantly greater compared to the TriPort and GelPOINT, and wrist extension for CL was significantly less compared to the TriPort and GelPOINT (Figure 5). Although the SILS Port failed to reach statistical significance, it exhibits a similar trend to the other single-port devices.

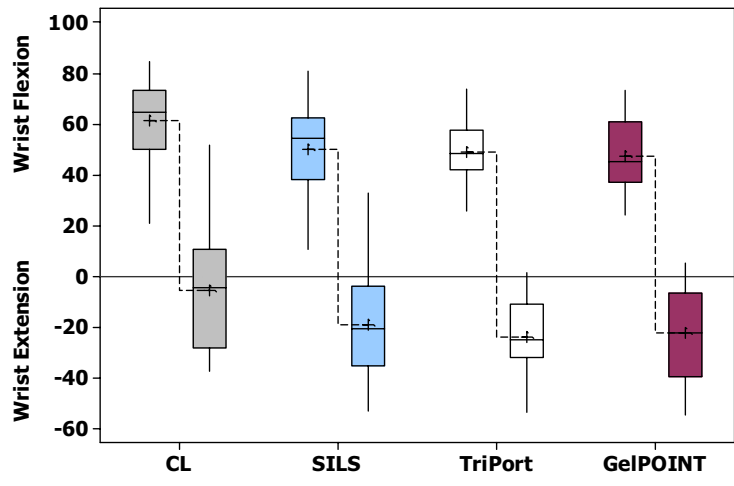

Figure 5. Wrist Flexion/Extension Notes: median horizontal line, mean plus sign, mean ROM dashed line

\subsection{Wrist radial/ulnar deviation}

Average wrist ROM, radial deviation and ulnar deviation were significantly different across all ports $(\mathrm{p}<0.05)$. CL had significantly less ROM at $31^{\circ}$ compared to the SILS Port at $52^{\circ}$, TriPort at $47^{\circ}$ and GelPOINT at $44^{\circ}$ (Figure 6). ROM was also significantly less for the GelPOINT compared to the SILS Port. Second, ulnar deviation was significantly less for CL compared to the single-port devices. Lastly, radial deviation was significantly less for the TriPort and GelPOINT at $10^{\circ}$ compared to both CL and SILS Port at $20^{\circ}$.

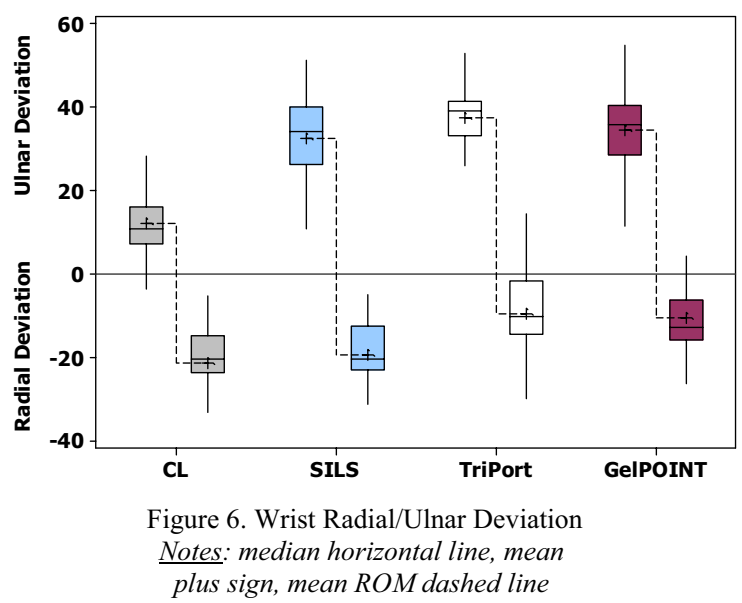




\subsection{Comfort/discomfort}

Laparoscopic instruments have been associated with nerve injury and neuropraxia of the digits, most often the thumb, due to handle design and gripping techniques [3]. Accordingly, it was hypothesized that the thumb, index and middle fingers may experience greater discomfort due to the novel LESS instrumentation. In general there was very little variation between median comfort/discomfort ratings, resulting in no significant differences across ports for all six anatomical regions (Table 2). In general, participants were more likely to agree that CL was comfortable and that the single-port devices were comfortable or slightly comfortable for all regions. Overall, there was no significant difference between CL and LESS based on subjective comfort/discomfort ratings of the thumb, index and middle fingers, palm, forearm and upper arm.

Table 2

Subjective Comfort Assessments

\begin{tabular}{|r|c|c|c|c|}
\hline & $\begin{array}{c}\text { CL } \\
\text { Ports }\end{array}$ & $\begin{array}{c}\text { SILS } \\
\text { Port }\end{array}$ & TriPort & $\begin{array}{c}\text { Gel- } \\
\text { POINT }\end{array}$ \\
\hline Thumb & $\begin{array}{c}5.0 \\
(1.0)\end{array}$ & $\begin{array}{c}4.5 \\
(1.0)\end{array}$ & $\begin{array}{c}4.0 \\
(2.0)\end{array}$ & $\begin{array}{c}4.0 \\
(2.0)\end{array}$ \\
\hline Index & 5.0 & 5.0 & 5.0 & 5.0 \\
Finger & $(1.0)$ & $(0.75)$ & $(0.0)$ & $(0.75)$ \\
\hline Middle & 5.0 & 5.0 & 5.0 & 5.0 \\
Finger & $(1.0)$ & $(1.0)$ & $(1.75)$ & $(1.0)$ \\
\hline Palm & 5.0 & 5.0 & 5.0 & 5.0 \\
& $(0.75)$ & $(0.75)$ & $(1.75)$ & $(1.0)$ \\
\hline Forearm & 5.0 & 5.0 & 5.0 & 5.0 \\
& $(2.0)$ & $(1.5)$ & $(1.75)$ & $(1.0)$ \\
\hline Upper & 5.0 & 5.0 & 5.0 & 5.0 \\
Arm & $(1.0)$ & $(1.75)$ & $(.02)$ & $(1.0)$ \\
\hline
\end{tabular}

Notes: Median (Interquartile Range), 1-Very Uncomfortable, 2-Uncomfortable, 3-Slightly Uncomfortable, 4-Slightly Comfortable, 5-Comfortable, and 6-Very Comfortable

\section{Discussion}

Based solely on the physical comfort/discomfort ratings, there appears to be no difference between conventional laparoscopy and LESS. However, the simplified task and novice participants may have affected these results. Similarly, range of motion for both elbow and wrist flexion/extension were similar for conventional laparoscopy and the single-port devices. However, upon closer inspection wrist flexion/extension for the single port devices was occur- ring with less flexion and more extension compared to conventional laparoscopy. Additionally, wrist radi$\mathrm{al} /$ ulnar deviation and range of motion were dramatically different between conventional laparoscopy and the single-port devices. Presumably the close proximity of the instruments, restrictive nature of the singleport device, and the need to achieve adequate instrument triangulation and visualization for LESS resulted in greater wrist deviation and range of motion. Since the wrist is one of the most common joints to be affected by cumulative trauma disorders (CTDs) [13], the added strain on the wrist during LESS may result in a new occupational hazard for laparoscopic surgeons. However, these preliminary findings will need to be confirmed in order to determine the likelihood and severity of injury. Overall, further investigation of the ergonomics of LESS is needed in order to better train and equip laparoendoscopic single-site surgeons for optimal performance.

Study limitations include the inclusion of only novice trainees. Future studies will include participants with different levels of surgical experience as well as more difficult training tasks. In addition, bent and articulating instruments are currently being used during many LESS procedures and will also be included in future studies. Finally, since the participants were novices, there may have been effects due to fatigue and learning.

\section{Acknowledgements}

The authors wish to acknowledge the members of the University of Nebraska Medical Center's Center for Advanced Surgical Technology (CAST), Sorrell Center for Health Science Education Clinical Skill Laboratory, and Innovative Design and Ergonomic Analysis (IDEA) Laboratory for their assistance in this study. The authors would also like to acknowledge the Nebraska Research Initiative for partial support of this study.

\section{Disclosure}

Authors B. McCrory, B.R. Lowndes, L.M. Wirth, A.E. de Laveaga, C.A. LaGrange, and M.S. Hallbeck have no conflicts of interest or financial ties to disclose. 


\section{References}

[1] E.D. Auyang, B.F. Santos, D.H. Enter, E.S. Hungness and N.J. Soper, Natural orifice translumenal endoscopic surgery (NOTES): A technical review, Surg Endosc 25 (2010), 3135 48.

[2] B. Brown-Clerk, A.E. de Laveaga, C.A. LaGrange, L.M Wirth, B.R. Lowndes and M.S. Hallbeck, Laparoendoscopic single-site (LESS) surgery versus conventional laparoscopic surgery: comparison of surgical port performance in a surgical simulator with novices, Surg Endosc 25 (2011), 2210-8.

[3] U. De, Ergonomics and Laparoscopy, Indian J Surg 67 (2005), 164-6.

[4] A.M. Derossis, G.M. Fried, M. Abrahamowicz, H.H. Sigman, J.S. Barkun and J.L. Meakins, Development of a model for training and evaluation of laparoscopic skills, American Journal of Surgery, 175(1998), 482-487.

[5] G.M. Fried, A.M. Derossis, J. Bothwell and H.H. Sigman, Comparison of laparoscopic performance in vivo with performance measured in a laparoscopic simulator. Surg Endosc 13(1999), 1077-1081.

[6] M.T. Gettman, W.M. White, M. Aron, R. Autorino, T. Averch, G. Box, et al., Endourological Society NOTES and LESS Working Group, European Society of Urotechnology NOTES and LESS Working Group. Where do we really stand with LESS and NOTES? Eur Urol. 59 (2011), 231-4.

[7] I.S. Gill, A.P. Advincula, M. Aron, J. Caddedu, D. Canes, P.G. Curcillo, 2nd, et al., Consensus statement of the consortium for laparoendoscopic single-site surgery, Surg Endosc 24 (2009), 762-768.
[8] U. Matern and S. Koneczny, Safety, hazards and ergonomics in the operating room, Surg Endosc 21(2007), 1965-1969.

[9] A. Park, G. Lee, F.J. Seagull, N. Meenaghan and D. Dexter, Patients benefit while surgeons suffer: An impending epidemic. Journal of the American College of Surgeon, 210 (2010), 306-313.

[10]H. Rivas, E. Varela and D. Scott, Single-incision laparoscopic cholecystectomy: Initial evaluation of a large series of patients. Surg Endosc 24 (2010), 1403-12.

[11]A.A. Saber, M.H. Elgamal, T.H. El-Ghazaly, A.V. Dewoolkar, and A. Akl, Simple technique for single incision transumbilical laparoscopic appendectomy. International Journal of Surgery (London, England) 8 (2010), 128-130.

[12] K. Slim and M.V. Launay-Savary, NOTES, the debate continues. Surg Endosc. 22 (2008): 2326-7.

[13]S. Tanaka, P. Seligman, W. Halperin, M. Thun, C.M. Timbrook and J.J. Wasil, Use of workers compensation claims data for surveillance of cumulative trauma disorders (CTDs). J. Occup. Med. 30 (1988), 488-492.

[14]J. Teixeira, K. McGill, N. Koshy, J. McGinty and G. Todd, Laparoscopic single-site surgery for placement of adjustable gastric band--a series of 22 cases. Surgery for Obesity and Related Diseases: Official Journal of the American Society for Bariatric Surgery 6(2010), 41-5.

[15]M. van Det, W. Meijerink, C. Hoff, E. Totte and J. Pierie, Optimal ergonomics for laparoscopic surgery in minimally invasive surgery suites: A review and guidelines. Surgical Endoscopy, 23 (2009), 1279-85.

[16]M.A. van Veelen, J.J. Jakimowicz, G. Kazemier, Improved physical ergonomics of laparoscopic surgery. Minimally Invasive Ther. Allied Technol. 13 (2004), 161-6. 